\title{
The Ground State and Excitations of Spin Chain with Orbital Degeneracy
}

\author{
You-Quan $\mathrm{Li}^{1,2}$, Michael $\mathrm{Ma}^{1}$, Da-Ning $\mathrm{Shi}^{1,3}$, and Fu-Chun Zhang ${ }^{1}$ \\ 1 Department of Physics, University of Cincinnati, Cincinnati OH 45221 \\ ${ }^{2}$ Zhejiang Institute of Modern Physics, Zhejiang University, Hangzhou 310027, China \\ ${ }^{3}$ College of Science, Nanjing Univ. of Aeronautics and Astronautics, Nanjing, China
}

(Received Feb. 16, 1999)

\begin{abstract}
The one dimensional Heisenberg model in the presence of orbital degeneracy is studied at the $S U(4)$ symmetric point by means of Bethe ansatz. Following Sutherland's previous work on an equivalent model, we discuss the ground state and the low-lying excitations more extensively in connection to the spin systems with orbital degeneracy. We show explicitly that the ground state is a $S U(4)$ singlet. We study the degeneracies of the elementary excitations and the spectra of the generalized magnons consisting of these excitations. We also discuss the complex 2-strings in the context of the Bethe ansatz solutions.
\end{abstract}

PACS number(s): 75.10.-b, 75.10.Jm, 03.65.Ge, 74.20.Mn

\section{INTRODUCTION}

There has been much interest recently in spin Hamiltonians with orbital degeneracy. The orbital degree of freedom may be relevant to many transitional metal oxides [1 11]. Examples of such systems in 1-dimension include quasi-one-dimensional tetrahis-dimethylaminoethylene (TDAE)- $\mathrm{C}_{60}$ 12], and artificial quantum dot arrays [13. Recently, we discussed these systems 14] within the framework of a $S U(4)$ theory. A quantum disordered ground state in 2-dimension was proposed to be relevant to the experimentally observed unusual magnetic properties in $\mathrm{LiNiO}_{2}$. There have also been numerical studies of the 1-dimensional models for these systems [15, 16 .

In the present paper, we use the Bethe ansatz method to study the $S U(4)$ symmetric Heisenberg spin chain with two-fold orbital degeneracy. This model is equivalent to the model studied by Pokrovskii and Uimin [17], and to one of a class of models that has been solved by Sutherland [18]. Expanding on Sutherland's work, we study the ground state and low-lying excitations more extensively by considering holes and 2-strings in the thermodynamics limit, and in connection to the spin systems with orbital degeneracy. We show explicitly that the ground state is a $S U(4)$ singlet, consistent with the generalized LiebMattis theorem of Affleck and Lieb [22]. We discuss the degeneracies of the elementary excitations and the spectra of the generalized magnons resulting from such such excitations. We also discuss the complex 2-strings in the context of the Bethe ansatz solutions. The paper is organized as follows. In section II, we introduce the model and discuss its symmetry. In section III, we present the Bethe ansatz solution following Sutherland's approach. We discuss the ground state in section IV, and the elementary excitations and the generalized magnon modes in section V. A brief summary is given in section VI.

\section{SYMMETRY CONSIDERATION}

We consider the spin chain of $\mathrm{N}$ sites with two-fold orbital-degeneracy and with periodic boundary condition [6]

$$
\mathcal{H}=\sum_{j=1}^{N} J\left[\left(2 \vec{T}_{j} \cdot \vec{T}_{j+1}+\frac{1}{2}\right)\left(2 \vec{S}_{j} \cdot \vec{S}_{j+1}+\frac{1}{2}\right)-1\right],
$$

where $\vec{S}_{j}$ and $\vec{T}_{j}$ are the spin and orbital operators respectively on the $j$-th site, and are each generators of a $S U(2)$ Lie algebra. Clearly, in addition to the permutation symmetry $(H$ is invariant under the transformation $j \rightarrow j+1$ for all $\left.j^{\prime} s\right)$, the system has an explicit $S U(2) \otimes S U(2)$ global symmetry and a bi-symmetry $\vec{S}_{j} \leftrightarrow \vec{T}_{j}$. Actually the Hamiltonian (11) is invariant under a global $S U(4)$ transformation, which is generated by $S^{\alpha}=\sum_{j} S_{j}^{\alpha}$, $T^{\alpha}=\sum_{j} T_{j}^{\alpha}$, and $Y^{\alpha \beta}=\sum_{j} T_{j}^{\alpha} \otimes S_{j}^{\beta}(\alpha, \beta=1,2,3)$. These operators satisfy the following commutation relations

$$
\begin{aligned}
{\left[S^{\alpha}, S^{\beta}\right] } & =i \epsilon^{\alpha \beta \gamma} S^{\gamma}, \quad\left[S^{\alpha}, T^{\beta}\right]=0, \\
{\left[T^{\alpha}, T^{\beta}\right] } & =i \epsilon^{\alpha \beta \gamma} T^{\gamma},\left[T^{\alpha}, Y^{\beta \delta}\right]=i \epsilon^{\alpha \beta \gamma} Y^{\gamma \delta}, \\
{\left[S^{\alpha}, Y^{\delta \beta}\right] } & =i \epsilon^{\alpha \beta \gamma} Y^{\delta \gamma} \\
{\left[Y^{\alpha \mu}, Y^{\beta \nu}\right] } & =i \epsilon^{\alpha \beta \gamma} \delta^{\mu \nu} S^{\gamma}+i \epsilon^{\mu \nu \rho} \delta^{\alpha \beta} T^{\rho} .
\end{aligned}
$$

Such a symmetry was noticed by Wigner in the study of nuclei long time ago [19]. Since the group $S U(4)$ is of rank three, there are three conserved quantum numbers in general. It is useful to write the above commutation relations in terms of Chevalley basis, i.e., three generators in the Cartan sub-algebra of $S U(4)$ (precisely, the $A_{3}$ Lie algebra) $H_{n}=\sum_{j=1}^{N} H_{n}(j)(n=1,2,3)$ which can be diagonalised simultaneously, and the other twelve generators $E_{\alpha}=\sum_{j=1}^{N} E_{\alpha}(j)$ ( $\alpha$ denotes root vectors). The local generators $H_{n}(j)$ and $E_{\alpha}(j)$ are related to the spin and orbital operators by, 


$$
\begin{aligned}
H_{1}(j)= & \left(1+2 T_{j}^{z}\right) S_{j}^{z}, \quad E_{\alpha_{1}}(j)=\left(\frac{1}{2}+T_{j}^{z}\right) S_{j}^{+}, \\
H_{2}(j)= & \left(T_{j}^{z}-S_{j}^{z}\right), \quad E_{\alpha_{2}}(j)=T_{j}^{+} S_{j}^{-}, \\
H_{3}(j)= & \left(1-2 T_{j}^{z}\right) S_{j}^{z}, \quad E_{\alpha_{3}}(j)=\left(\frac{1}{2}-T_{j}^{z}\right) S_{j}^{+}, \\
& E_{\alpha_{1}+\alpha_{2}}(j)=T_{j}^{+}\left(\frac{1}{2}+S_{j}^{z}\right), \\
& E_{\alpha_{2}+\alpha_{3}}(j)=T_{j}^{+}\left(\frac{1}{2}-S_{j}^{z}\right), \\
& E_{\alpha_{1}+\alpha_{2}+\alpha_{3}}(j)=T_{j}^{+} S_{j}^{+},
\end{aligned}
$$

where $\alpha_{1}, \alpha_{2}$ and $\alpha_{3}$ denote the simple roots of $A_{3}$ Lie algebra [21]. The generators corresponding to the negative roots are given by $E_{-\alpha}(j)=E_{\alpha}^{\dagger}(j)$. One can verify that these operators satisfy the standard commutation relations of $A_{3}$ Lie algebra, and Eq.(11) can be rewritten as,

$$
\mathcal{H}=\sum_{j}[h(j, j+1)-3 / 4]
$$

$h(j, j+1)=\sum_{m, n} g^{m n} H_{m}(j) H_{n}(j+1)+\sum_{\alpha \in \Delta} E_{\alpha}(j) E_{-\alpha}(j+1)$, where $\Delta$ denotes the set of roots of the Lie algebra. We denote the spin components by up $(\uparrow)$ and down $(\downarrow)$, and the orbital components by top and bottom. Then the four possible states on each site are

$$
\begin{aligned}
& |1>:=| \stackrel{\uparrow}{\uparrow}>=\mid 1 / 2,1 / 2>, \\
& |2>:=| \underset{-}{\downarrow}>=\mid-1 / 2,1 / 2>, \\
& |3>:=| \bar{\uparrow}>=\mid 1 / 2,-1 / 2>, \\
& |4>:=| \bar{\downarrow}>=\mid-1 / 2,-1 / 2>.
\end{aligned}
$$

The local lowering/raising operators $E_{ \pm \alpha_{n}}(j)$ relate those four states on the $j$ th site as follows,

$$
\begin{aligned}
E_{-\alpha_{n}}(j) \mid n>_{j} & \rightarrow \mid n+1>_{j}, \\
E_{\alpha_{n}}(j) \mid n+1>_{j} & \rightarrow \mid n>_{j}, n=1,2,3 .
\end{aligned}
$$

In terms of those operators, a general state can be written as

$$
\left.\left|\psi>=\sum_{\left\{x_{i}\right\}\left\{y_{j}\right\}\left\{z_{k}\right\}} \psi(x ; y ; z) \prod_{k=1}^{M^{\prime \prime}} E_{-\alpha_{3}}\left(z_{k}\right) \prod_{j=1}^{M^{\prime}} E_{-\alpha_{2}}\left(y_{j}\right) \prod_{i=1}^{M} E_{-\alpha_{1}}\left(x_{i}\right)\right| \uparrow \stackrel{\uparrow}{-} \cdots \stackrel{\uparrow}{-}\right\rangle
$$

where $x:=\left(x_{1}, x_{2}, \cdots, x_{M}\right), y:=\left(y_{1}, y_{2}, \cdots, y_{M^{\prime}}\right)$, $z:=\left(z_{1}, z_{2}, \cdots, z_{M^{\prime \prime}}\right) ; 1 \leq x_{1}<x_{2}<\cdots<x_{M} \leq N$; $x_{1} \leq y_{1}<y_{2}<\cdots<y_{M^{\prime}} \leq x_{M}, y_{1} \leq z_{1}<z_{2}<$ $\cdots<z_{M^{\prime \prime}} \leq y_{M^{\prime}}$; and $\left\{z_{k}\right\} \subset\left\{y_{j}\right\} \subset\left\{x_{i}\right\}$. We may define the weights as the eigenvalues of the global operator $H_{n}$, indicated by $\left(H_{1}, H_{2}, H_{3}\right)$. The eigenvalues of the local operators $H_{1}(j), H_{2}(j), H_{3}(j)$ acting on the four local states $\left|\stackrel{\uparrow}{-}>_{j},\right|_{-}^{\downarrow}>_{j}, \mid \bar{\uparrow}>_{j}$ and $\mid \bar{\downarrow}>_{j}$ are $(1,0,0)$, $(-1,1,0),(0,-1,1)$ and $(0,0,-1)$ respectively. We shall focus on the state with the highest weight. The other states in the same irreducible representation can then be obtained by using the corresponding lowering operators $E_{-\alpha_{n}}$. In the present model, the irreducible representation of the $S U(4)$ group of a N-site system is labeled by

$$
\left(N+M^{\prime}-2 M, M+M^{\prime \prime}-2 M^{\prime}, M^{\prime}-2 M^{\prime \prime}\right) .
$$

\section{THE BETHE ANSATZ SOLUTION}

The permutation and the $S U(4)$ symmetries in the Hamiltonian enable us to seek the eigenstate of both the cyclic permutation operator and the generators of the Cartan subalgebra of $A_{3}$. The invariance of the cyclic permutation imposes a periodic boundary condition on the wave function $\psi(x, y, z)$. The present model is solvable [18], and the Bethe ansatz equations for the spectra are

$$
\begin{aligned}
\left(\frac{\lambda_{j}+i / 2}{\lambda_{j}-i / 2}\right)^{N} & =-\prod_{l=1}^{M} \frac{\lambda_{j}-\lambda_{l}+i}{\lambda_{j}-\lambda_{l}-i} \prod_{\beta=1}^{M^{\prime}} \frac{\mu_{\beta}-\lambda_{j}+i / 2}{\mu_{\beta}-\lambda_{j}-i / 2}, \\
\prod_{l=1}^{M} \frac{\mu_{\gamma}-\lambda_{l}+i / 2}{\mu_{\gamma}-\lambda_{l}-i / 2} & =-\prod_{\beta=1}^{M^{\prime}} \frac{\mu_{\gamma}-\mu_{\beta}+i}{\mu_{\gamma}-\mu_{\beta}-i} \prod_{b=1}^{M^{\prime \prime}} \frac{\nu_{b}-\mu_{\gamma}+i / 2}{\nu_{b}-\mu_{\gamma}-i / 2}, \\
\prod_{\beta=1}^{M^{\prime}} \frac{\nu_{c}-\mu_{\beta}+i / 2}{\nu_{c}-\mu_{\beta}-i / 2} & =-\prod_{b=1}^{M^{\prime \prime}} \frac{\nu_{c}-\nu_{b}+i}{\nu_{c}-\nu_{b}-i},
\end{aligned}
$$

where $j, l=1,2, \cdots, M ; \beta, \gamma=1,2, \cdots, M^{\prime}$ and $b, c=$ $1,2, \cdots, M^{\prime \prime}$. These are secular equations for the spectra of $S U(4)$ rapidities $\lambda, \mu$ and $\nu$. The energy spectrum is given by

$$
E=-\sum_{l=1}^{M} \frac{J}{(1 / 2)^{2}+\lambda_{l}^{2}}
$$


The momentum defined by the translation of the system along the chain is given by

$$
\begin{aligned}
P & =\frac{1}{i} \ln \prod_{l=1}^{M} \frac{\lambda_{l}+i / 2}{\lambda_{l}-i / 2} \\
& =\sum_{l=1}^{M}\left[\pi-2 \tan ^{-1}\left(2 \lambda_{l}\right)\right] .
\end{aligned}
$$

Note that $P$ in eq. (9) is determined up to $\bmod (2 \pi)$, and the inverse trigonometric function is defined in the main branch. We have included explicitly the $\pi$ term in eq. (9), which is usually neglected in the study of pure spin Heisenberg models 20]. In the $S U(4)$ model, there are three types of elementary excitations as we will discuss below, and it is convenient to include the $\pi$ term in $P$ to study the magnon types of composite excitations. We define the momentum of the elementary excitations as the momentum relative to the ground state [20]. By taking the logarithm of (7), a set of coupled transcendental equations are obtained,

$$
\begin{array}{r}
\Theta_{1 / 2}\left(\lambda_{j}\right)-\frac{1}{N} \sum_{l=1}^{M} \Theta_{1}\left(\lambda_{j}-\lambda_{l}\right)-\frac{1}{N} \sum_{\beta}^{M^{\prime}} \Theta_{1 / 2}\left(\mu_{\beta}-\lambda_{j}\right)=\frac{2 \pi}{N} I_{j}, \\
\sum_{l=1}^{M} \Theta_{1 / 2}\left(\mu_{\gamma}-\lambda_{l}\right)-\sum_{\beta=1}^{M^{\prime}} \Theta_{1}\left(\mu_{\gamma}-\mu_{\beta}\right)-\sum_{b=1}^{M^{\prime \prime}} \Theta_{1 / 2}\left(\nu_{b}-\mu_{\gamma}\right)=2 \pi J_{\gamma}, \\
\sum_{\beta=1}^{M^{\prime}} \Theta_{1 / 2}\left(\nu_{c}-\mu_{\beta}\right)-\sum_{b=1}^{M^{\prime \prime}} \Theta_{1}\left(\nu_{c}-\nu_{b}\right)=2 \pi K_{c},
\end{array}
$$

where $\Theta_{\rho}(x):=2 \tan ^{-1}(x / \rho)$. The quantum number $I_{j}$ is an integer or half-integer depending on whether $N-M-M^{\prime}$ is odd or even, and so is $J_{\gamma}\left(\right.$ or $\left.K_{c}\right)$ depending on whether $M-M^{\prime}-M^{\prime \prime}$ (or $M^{\prime}-M^{\prime \prime}$ ) is odd or even. These properties arise from the logarithm function.

Replacing $\lambda_{j}, \mu_{\gamma}$ and $\nu_{c}$ in eq. (10) by continuous variables $\lambda, \mu$ and $\nu$ but keeping the summation still over the solution set of these roots $\left\{\lambda_{l}, \mu_{\beta}, \nu_{b}\right\}$, we can consider the quantum numbers $I_{j}, J_{\gamma}$ and $K_{c}$ as functions $I(\lambda), J(\mu)$ and $K(\nu)$ given by eq. (10). Take $I(\lambda)$ as an example. When $I(\lambda)$ passes through one of the quantum numbers $I_{j}$, the corresponding $\lambda$ is equal to one of the roots $\lambda_{j}$. Similarly for $J(\mu)$ or $K(\nu)$. However, there may exist some integers or half-integers for which the corresponding $\lambda(\mu$ or $\nu)$ is not in the set of roots. We shall name such a state as a "hole". In the thermodynamics limit $N \rightarrow \infty$, we may introduce the density of roots and the density of holes (indicated by a subscript $h$ ),

$$
\begin{aligned}
\sigma(\lambda)+\sigma_{h}(\lambda) & =(1 / N) d I(\lambda) / d \lambda \\
\omega(\mu)+\omega_{h}(\mu) & =(1 / N) d J(\mu) / d \mu \\
\tau(\nu)+\tau_{h}(\nu) & =(1 / N) d K(\nu) / d \nu
\end{aligned}
$$

By replacing the summations by integrals,

$$
\begin{array}{r}
\lim _{N \rightarrow \infty} \frac{1}{N} \sum_{l=1}^{M} f\left(\lambda_{l}\right)=\int_{-B}^{B} d \lambda \sigma(\lambda) f(\lambda), \\
\lim _{N \rightarrow \infty} \frac{1}{N} \sum_{\beta=1}^{M^{\prime}} f\left(\mu_{\beta}\right)=\int_{-B^{\prime}}^{B^{\prime}} d \mu \omega(\mu) f(\mu), \\
\lim _{N \rightarrow \infty} \frac{1}{N} \sum_{b=1}^{M^{\prime \prime}} f\left(\nu_{b}\right)=\int_{-B^{\prime \prime}}^{B^{\prime \prime}} d \nu \tau(\nu) f(\nu),
\end{array}
$$

eq.(9) become the coupled integral equations,

$$
\begin{aligned}
& \sigma(\lambda)+\sigma_{h}(\lambda)=K_{1 / 2}(\lambda)-\int_{-B}^{B} d \lambda^{\prime} K_{1}\left(\lambda-\lambda^{\prime}\right) \sigma\left(\lambda^{\prime}\right)+\int_{-B^{\prime}}^{B^{\prime}} d \mu^{\prime} K_{1 / 2}\left(\lambda-\mu^{\prime}\right) \omega\left(\mu^{\prime}\right), \\
& \omega(\mu)+\omega_{h}(\mu)=\int_{-B}^{B} d \lambda^{\prime} K_{1 / 2}\left(\mu-\lambda^{\prime}\right) \sigma\left(\lambda^{\prime}\right)-\int_{-B^{\prime}}^{B^{\prime}} d \mu^{\prime} K_{1}\left(\mu-\mu^{\prime}\right) \omega\left(\mu^{\prime}\right)+\int_{-B^{\prime \prime}}^{B^{\prime \prime}} d \nu^{\prime} K_{1 / 2}\left(\mu-\nu^{\prime}\right) \tau\left(\nu^{\prime}\right), \\
& \tau(\nu)+\tau_{h}(\nu)=\int_{-B^{\prime}}^{B^{\prime}} d \mu^{\prime} K_{1 / 2}\left(\nu-\mu^{\prime}\right) \omega\left(\mu^{\prime}\right)-\int_{-B^{\prime \prime}}^{B^{\prime \prime}} d \nu^{\prime} K_{1}\left(\nu-\nu^{\prime}\right) \tau\left(\nu^{\prime}\right),
\end{aligned}
$$


where $K_{\rho}(x):=\pi^{-1} \rho /\left(\rho^{2}+x^{2}\right)$, and $B, B^{\prime}$, and $B^{\prime \prime}$ in the definite integrals should be determined self-consistently. In the absence of the complex roots, $M / N=\int_{-B}^{B} \sigma(\lambda) d \lambda$, $M^{\prime} / N=\int_{-B^{\prime}}^{B^{\prime}} \omega(\mu) d \mu$, and $M^{\prime \prime} / N=\int_{-B^{\prime \prime}}^{B^{\prime \prime}} \tau(\nu) d \nu$. Once the density $\sigma$ is solved from eq.(11), we have the $z$ components of the total spin and the total orbital

$$
\begin{aligned}
\frac{S_{\text {tot }}^{z}}{N}=\frac{1}{2}+\int_{-B^{\prime}}^{B^{\prime}} \omega(\mu) d \mu-\int_{-B}^{B} \sigma(\lambda) d \lambda-\int_{-B^{\prime \prime}}^{B^{\prime \prime}} \tau(\nu) d \nu, \\
\frac{T_{\text {tot }}^{z}}{N}=\frac{1}{2}-\int_{-B^{\prime}}^{B^{\prime}} \omega(\mu) d \mu,
\end{aligned}
$$

the energy

$$
E=-2 \pi N J \int_{-B}^{B} K_{1 / 2}(\lambda) \sigma(\lambda) d \lambda,
$$

and the momentum

$$
P=-N \int_{-B}^{B}\left[2 \tan ^{-1}(2 \lambda)-\pi\right] \sigma(\lambda) d \lambda .
$$

\section{THE GROUND STATE}

The ground state is described by the densities $\sigma_{0}(\lambda)$, $\omega_{0}(\mu)$, and $\tau_{0}(\nu)$ with no holes and by $B_{0}=B_{0}^{\prime}=B_{0}^{\prime \prime} \rightarrow$ $\infty$. This is true because all the states with holes will have higher energies. In this case, eq. (11) can be solved. Let

$$
\begin{aligned}
& \sigma_{0}(\lambda)=\frac{1}{2 \pi} \int_{-\infty}^{\infty} \tilde{\sigma}_{0}(q) e^{-i q \lambda} d q, \\
& \omega_{0}(\mu)=\frac{1}{2 \pi} \int_{-\infty}^{\infty} \tilde{\omega}_{0}(q) e^{-i q \mu} d q, \\
& \tau_{0}(\nu)=\frac{1}{2 \pi} \int_{-\infty}^{\infty} \tilde{\tau}_{0}(q) e^{-i q \nu} d q,
\end{aligned}
$$

then

$$
\begin{aligned}
\tilde{\sigma}_{0}(q) & =\sinh (3 q / 2) / \sinh (2 q), \\
\tilde{\omega}_{0}(q) & =\sinh (q) / \sinh (2 q), \\
\tilde{\tau}_{0}(q) & =\sinh (q / 2) / \sinh (2 q) .
\end{aligned}
$$

Hence from eq. (6), the highest weight labeling the ground state is the null vector $(0,0,0)$, and so the ground state is a $S U(4)$ singlet. This agrees to the theorem of Affleck and Lieb 22], a generalization of the spin chain problem 23]. In that state, the total orbital, the total spin, and their products are all zero, i.e.,

$$
\begin{aligned}
& \frac{T_{t o t}^{z}}{N}=\frac{1}{2}-\int_{-\infty}^{\infty} \omega_{0}(\mu) d \mu=\frac{1}{2}-\tilde{\omega}_{0}(0)=0, \\
& \frac{S_{t o t}^{z}}{N}=\frac{1}{2}+\tilde{\omega}_{0}(0)-\tilde{\sigma}_{0}(0)-\tilde{\tau}_{0}(0)=0, \\
& \sum_{j=1}^{N} T_{j}^{z} S_{j}^{z}=0 .
\end{aligned}
$$

In deriving this result, eqs.(3) have been used. The energy and the momentum of the ground state are

$$
\begin{aligned}
& E_{0}=-N J\left(\frac{3}{2} \ln 2+\frac{\pi}{4}\right) \\
& P_{0}= \begin{cases}0 \bmod 2 \pi, & \text { for } N / 4=\text { even }, \\
\pi \bmod 2 \pi, & \text { for } N / 4=\text { odd } .\end{cases}
\end{aligned}
$$

Eq. (13) coincides with the result of Sutherland [18 after correcting for the trivial overall constant shift $J N$ between the two models.

\section{LOW-LYING EXCITATIONS}

\section{A. Spectra of elementary excitations}

The possible elementary excitation modes are obtained by the variation in the sequence of quantum numbers $\left\{I_{j}\right\},\left\{J_{\gamma}\right\}$ or $\left\{K_{c}\right\}$ from the ground state. We can assume $B=B^{\prime}=B^{\prime \prime} \rightarrow \infty$ for the low-lying excitations. The simple modes will be solved by placing holes in the rapidity-configurations. If we let $\sigma(\lambda)=$ $\sigma_{0}(\lambda)+\sigma_{1}(\lambda) / N, \omega(\mu)=\omega_{0}(\mu)+\omega_{1}(\mu) / N$ and $\tau(\nu)=$ $\tau_{0}(\nu)+\tau_{1}(\nu) / N$, then the excitation energy and momentum

$$
\begin{array}{r}
\Delta E=-2 \pi J \int_{-\infty}^{\infty} K_{1 / 2}(\lambda) \sigma_{1}(\lambda) d \lambda, \\
\Delta P=-\int_{-\infty}^{\infty}\left[2 \tan ^{-1}(2 \lambda)-\pi\right] \sigma_{1}(\lambda) d \lambda,
\end{array}
$$

and $\Delta M=\int \sigma_{1}(\lambda) d \lambda, \Delta M^{\prime}=\int \omega_{1}(\mu) d \mu$, and $\Delta M^{\prime \prime}=$ $\int \tau_{1}(\nu) d \nu$. After solving the integral equations (11) with $\sigma_{h}(\lambda)=\delta(\lambda-\bar{\lambda}) / N, \omega_{h}(\mu)=\delta(\mu-\bar{\mu}) / N$ or $\tau_{h}(\nu)=\delta(\nu-\bar{\nu}) / N$ respectively, one finds that there are three types of elementary excitation modes. A hole in the $\lambda$-configuration, $\mu$-configuration, or $\nu$-configuration ( $\lambda$-hole, $\mu$-hole, and $\nu$-hole respectively hereafter) creates a $S U(4)$ multiplet labeled by the highest weight $(1,0,0)$, $(0,1,0)$ or $(0,0,1)$ respectively. The $\mu$-hole has a six dimensional representation, the $\lambda$ - and $\nu$-holes have four dimensional representations. We call these excitations the $S U(4)$ flavorons. The energies of these elementary excitation are

$$
\begin{aligned}
\varepsilon_{\sigma}(\bar{\lambda}) & =\frac{J \pi / 2}{\sqrt{2} \cosh (\bar{\lambda} \pi / 2)-1}, \\
\varepsilon_{\omega}(\bar{\mu}) & =\frac{J \pi / 2}{\cosh (\bar{\mu} \pi / 2)}, \\
\varepsilon_{\tau}(\bar{\nu}) & =\frac{J \pi / 2}{\sqrt{2} \cosh (\bar{\nu} \pi / 2)+1},
\end{aligned}
$$

where $\bar{\lambda}, \bar{\mu}$ and $\bar{\nu}$ stand for the positions of holes in the corresponding rapidity configurations. These excitation 
energies vanish when the positions of holes go to infinity in the thermodynamic limit. Therefore they are gapless modes. The momenta of the excitations are given by

$$
\begin{aligned}
& p_{\sigma}(\bar{\lambda})=2 \tan ^{-1}[(\sqrt{2}+1) \tanh (\bar{\lambda} \pi / 4)]-3 \pi / 4, \\
& p_{\omega}(\bar{\mu})=2 \tan ^{-1}[\tanh (\bar{\mu} \pi / 4)]-\pi / 2, \\
& p_{\tau}(\bar{\nu})=2 \tan ^{-1}[(\sqrt{2}-1) \tanh (\bar{\nu} \pi / 4)]-\pi / 4
\end{aligned}
$$

Eliminating the rapidities in eqs. (15) and (16), we have

$$
\begin{aligned}
& \varepsilon_{\sigma}\left(p_{\sigma}\right)=\frac{J \pi}{2}\left[\sqrt{2} \cos \left(p_{\sigma}+3 \pi / 4\right)+1\right], \\
& \varepsilon_{\omega}\left(p_{\omega}\right)=\frac{J \pi}{2} \cos \left(p_{\omega}+\pi / 2\right), \\
& \varepsilon_{\tau}\left(p_{\tau}\right)=\frac{J \pi}{2}\left[\sqrt{2} \cos \left(p_{\tau}+\pi / 4\right)-1\right],
\end{aligned}
$$

where $p_{\sigma} \in[-3 \pi / 2,0], p_{\omega} \in[-\pi, 0], p_{\tau} \in[-\pi / 2,0]$.

We are now in the position to relate the elementary excitations to the spin and orbital in the original model, eq. (1). The quadruplets $(1,0,0)$ or $(0,0,1)$ are flavorons carrying both spin $1 / 2$ and orbital $1 / 2$ with energies $\epsilon_{\sigma}$ or $\epsilon_{\tau}$, the hexaplet $(0,1,0)$ describes flavoron $\mathrm{s}$ carrying either spin 1 or orbital 1 with energy $\epsilon_{\omega}$. The spectra of these three types of excitations are plotted in fig.(1). In comparison to Sutherland's results, eq.(17) differs only in that each mode is shifted by a different constant in momentum arising from the $\pi$ term in Eq. (9). It seems more convenient to use the present version, eq. (17), to study the spectra of the magnon-type excitations in the subsection V.C.

\section{B. The complex roots}

Because of the existance of the complex roots 25] in the solution set of the Bethe ansatz equations (7), we must consider their contributions, particularly from the 2 -strings. In this case we need to rederive the integral equations (11). We obtain the same equation formally but now the inhomogeneous terms $\sigma_{h}(\lambda), \omega_{h}(\mu)$, and $\tau_{h}(\nu)$ include also the contributions from the complex roots. A 2 -string in the $\lambda$-configuration, $\lambda_{ \pm}=\lambda_{0} \pm i / 2$, introduces additional terms in eq. (11). As a result, we have

$$
\begin{aligned}
\sigma_{h}(\lambda) & =\left[K_{3 / 2}\left(\lambda-\lambda_{0}\right)+K_{1 / 2}\left(\lambda-\lambda_{0}\right)\right] / N \\
\omega_{h}(\mu) & =-K_{1}\left(\mu-\lambda_{0}\right) / N \\
\tau_{h}(\nu) & =0 .
\end{aligned}
$$

The energy is given by

$$
\begin{aligned}
\Delta E= & -2 \pi J \int_{-\infty}^{\infty} K_{1 / 2}(\lambda) \sigma_{1}(\lambda) d \lambda \\
& -2 \pi J\left[K_{1 / 2}\left(\lambda_{0}+i / 2\right)+K_{1 / 2}\left(\lambda_{0}-i / 2\right)\right],
\end{aligned}
$$

and the integral equation leads to

$$
\tilde{\sigma_{1}}(q)=-\exp \left(i q \lambda_{0}-|q| / 2\right)
$$

Our calculation shows a complete cancellation in eq.(19). Therefore a 2-string in the $\lambda$-configuration does not change the energy. We also find $M=N \int \sigma(\lambda) d \lambda+2$, where $\sigma(\lambda)$ is the density of the real roots.

Similar results are found for the 2 -strings in the $\nu$ - and $\mu$-configurations. The equations for a $\nu$-configuration are given by,

$$
\begin{aligned}
\sigma_{h}(\lambda) & =0, \\
\omega_{h}(\mu) & =-K_{1}\left(\mu-\nu_{0}\right) / N \\
\tau_{h}(\nu) & =\left[K_{3 / 2}\left(\nu-\nu_{0}\right)+K_{1 / 2}\left(\nu-\nu_{0}\right)\right] / N
\end{aligned}
$$

and $M^{\prime \prime}=N \int \tau(\nu) d \nu+2$. By solving $\sigma_{1}(\lambda)=0$, we obtain

$$
\Delta E=-2 \pi J \int K_{1 / 2} \sigma_{1}(\lambda) d \lambda=0
$$

And the equations for a 2 -string in the $\mu$-configuration are given by,

$$
\begin{aligned}
\sigma_{h}(\lambda) & =-K_{1}\left(\lambda-\mu_{0}\right) / N, \\
\omega_{h}(\mu) & =\left[K_{1 / 2}\left(\mu-\mu_{0}\right)+K_{3 / 2}\left(\mu-\mu_{0}\right)\right] / N, \\
\tau_{h}(\nu) & =-K_{1}\left(\nu-\mu_{0}\right) / N,
\end{aligned}
$$

and $M^{\prime}=N \int \omega(\mu) d \mu+2$, we obtain $\sigma_{1}(\lambda)=0$ and hence $\Delta E=0$. Although these three types of the 2-strings do not contribute to the energy, they do contribute to the quantum numbers of spin and orbital, and to the highest weight of the $S U(4)$ representations.

\section{Generalized magnon type excitations}

The flavorons discussed in the previous subsection are elementary excitations of the system. These flavorons may combine to form composite excitations similar to the magnon excitations in the 1-dimensional spin chain [26], which are of interest to experiments and numerical simulations. In such a construction, the structure of the decomposition of the direct product of the $S U(4)$ fundamental representation must be taken into account. Let us consider $N=4 n$, the decomposition brings about a direct sum of a series of irreducible representations, i.e., $(0,0,0),(1,0,1),(0,2,0),(2,1,0),(4,0,0)$ etc. The composite excitation states include both the singlet $(0,0,0)$ and the multiplets of 15 -fold $(1,0,1)$, of 20 -fold $(0,2,0)$, and of 45 -fold $(2,1,0)$ or of 35 -fold $(4,0,0)$ etc. Those multifold excitations are the generalization of magnon excitations to the spin systems with orbital degeneracy. One $\lambda$-hole and one $\nu$-hole together create a 15 -fold multiplet with excitation energy and momentum,

$$
\begin{aligned}
& \Delta E_{(15)}=\varepsilon_{\sigma}(\bar{\lambda})+\varepsilon_{\tau}(\bar{\nu}), \\
& \Delta P_{(15)}=p_{\sigma}(\bar{\lambda})+p_{\tau}(\bar{\nu}),
\end{aligned}
$$


which is a pair of flavorons of $\sigma$-type and $\tau$-type. Two $\mu$-holes create a 20 -fold multiplet with

$$
\begin{aligned}
\Delta E_{(20)} & =\varepsilon_{\omega}\left(\bar{\mu}_{1}\right)+\varepsilon_{\omega}\left(\bar{\mu}_{2}\right), \\
\Delta P_{(20)} & =p_{\omega}\left(\bar{\mu}_{1}\right)+p_{\omega}\left(\bar{\mu}_{2}\right) .
\end{aligned}
$$

The 45-fold multiplet is a three-hole state created by two $\lambda$-holes and one $\mu$-hole, for which the excitation energy and momentum are

$$
\begin{aligned}
\Delta E_{(45)} & =\varepsilon_{\sigma}\left(\bar{\lambda}_{1}\right)+\varepsilon_{\sigma}\left(\bar{\lambda}_{2}\right)+\varepsilon_{\omega}(\bar{\mu}), \\
\Delta P_{(45)} & =p_{\sigma}\left(\bar{\lambda}_{1}\right)+p_{\sigma}\left(\bar{\lambda}_{2}\right)+p_{\omega}(\bar{\mu}) .
\end{aligned}
$$

Four $\lambda$-holes create a 35 -fold multiplet with

$$
\begin{aligned}
\Delta E_{(35)} & =\sum_{j=1}^{4} \varepsilon_{\sigma}\left(\bar{\lambda}_{j}\right), \\
\Delta P_{(35)} & =\sum_{j=1}^{4} p_{\sigma}\left(\bar{\lambda}_{j}\right) .
\end{aligned}
$$

The singlet excitation is obtained by placing a $\lambda$-hole, a $\nu$-hole and three 2 -strings in $\lambda, \mu$ and $\nu$-configurations respectively. The singlet is degenerate with the 15 -fold multiplet in energy, i.e.

$$
\Delta E_{(1)}=\varepsilon_{\sigma}(\bar{\lambda})+\varepsilon_{\tau}(\bar{\nu}) .
$$

In the above equations, $\epsilon_{\sigma}(\bar{\lambda}), \epsilon_{\omega}(\bar{\mu})$ and $\epsilon_{\tau}(\bar{\nu})$ are given by eq. (15), and $p_{\sigma}(\bar{\lambda}), p_{\omega}(\bar{\mu})$, and $p_{\tau}(\bar{\nu})$ are given by eq. (16).

The energy-momentum dispersion of various magnontypes of excitations are plotted in Fig. (2). In the spectrum calculations, we have used the periodicity in momentum $P$, so that $\Delta E(P+2 \pi)=\Delta E(P)$. For instance, for the 45 -fold degenerate states, $\Delta E_{(45)}(P)=$ $\epsilon_{\sigma}\left(q_{1}\right)+\epsilon_{\sigma}\left(q_{2}\right)+\epsilon_{\omega}\left(q_{3}\right)$, where $P=q_{1}+q_{2}+q_{3}$, with modula $2 \pi$. In a recent paper in Ref. 15 , the lower-lying excitations of model (11) were calculated numerically for finite systems. Their results are consistent with ours in Fig. 2. In particular, both the numerical calculations and the present Bethe ansatz solutions show the following feature: as the momentum $|p|$ increases from 0 to $\pi$, the lowest excitations are changed from the 15 -fold degenerate states to the 45 -fold degenerate states at $|P|=\pi / 2$.

In Fig. 3, we illustrate these generalized magnon types of composite low lying excited states. We start with a typical configuration of the the ground state in Fig. 3 (a), and the various generalized magnon excitations are created from the ground state by introducing two or more flavorons as shown in (b)-(f), which arise from various possible flips of spin or orbital or both. The flavoron indicated by the dashed box in fig.(3) moves in the background of the $S U(4)$ singlet carrying both energy and the quantum numbers. The $\sigma$-type (flavoron) excitation mode is a moving quaduplet with $\left.\right|_{-} ^{\uparrow}>$ being the local highest weight state.
The $\omega$-type excitation mode is a moving hexaplet with $|\stackrel{\uparrow}{\downarrow}>-| \underline{\downarrow} \underset{-}{\uparrow}>$ being the local highest weight state. The $\tau$-type excitation mode is a moving quaduplet with $|\stackrel{\uparrow}{-} \underset{\downarrow}{\downarrow}>-| \stackrel{\uparrow}{-\uparrow} \underline{\downarrow}>+\mid \underline{\downarrow}-\uparrow \stackrel{\uparrow}{-}>$

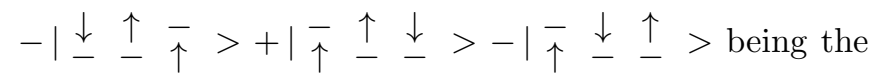
local highest weight state.

\section{SUMMARY AND ACKNOWLEDGMENT}

In this paper we have used Bethe ansatz method to discuss extensively the ground state and various types of the low lying excited states of a Heisenberg spin chain with two-fold orbital degeneracy in the limit of $S U(4)$ symmetry. There are three types of elementary excitations in the present model. Two of them carry spin $1 / 2$ and orbital $1 / 2$, and both are four-fold degenerate. The third one carries either spin 1 or orbital 1 , and is sixfold degenerate. We have constructed magnon types of composite excitations and calculated their spectra.

We thank G.S. Tian for a reference. YQL acknowledges grants NSFC-19675030, NSFZ-198024 and the support from the Y. Pao \& Z. Pao Foundation. The work is supported in part by DOE grant No. DE/FG0398ER45687.

[1] D. B. McWhan, T. M. Rice and J. P. Remeika, Phys. Rev. Lett 23, 1384 (1969); D. B. McWhan, A. Menth, J. P. Remeika, W. F. Brinkman and T. M. Rice, Phys. Rev. B7, 1920 (1973).

[2] C Castellani, C. R. Natoli and J. Ranninger, Phys. Rev. B18, 4945, 4967 and 5001 (1978).

[3] R. E. Word, S. A Werner, W. B. Yelon, J. M. Honig and S. Shivashankar, Phys. Rev. B23, 3533 (1981).

[4] K. I. Kugel and D. I. Khomskii, Usp. Fiz Nauk. 136, 621 (1982), [Sov. Phys. Ups. 25, 231 (1982)].

[5] W. Bao, C. Broholm, S. A. Carter, T. F. Rosenbaum, G. Aeppli, S. F. Trevino, P. Metcalf, J. M. Honig and J. Spalek, Phys. Rev. Lett. 71, 766 (1993).

[6] T. M. Rice, "Spectroscopy of Mott Insulators and Correlated Metals", Eds. A. Fujimori and Y. Tokura, Springer, Berlin (1995).

[7] H. F. Pen, J. ven den Brink, D. I. Komomskii, G. A. Sawatzky, Phys. Rev. Lett. 78, 1323 (1996).

[8] K. Yamaura, M. Takano, A. Hirano, R. Kanno, J. Solid State Chemistry 127, 109 (1997), and references therein.

[9] S. Ishihara, J. Inoue, and S. Maekawa, Phys. Rev. B55, 8280 (1997). S. Ishihara, M. Yamanaka, N. Nagaosa, Phys. Rev. B56, 686 (1997).

[10] R. Shiina, T. Nishitani, H. Shiba, J. Phys. Soc. Japan 66, 3159 (1997). 
[11] L. F. Feiner, A. M. Oles, and J. Zaanan, Phys. Rev. Lett. 78, 2799(1997).

[12] D. P. Arovas and A, Auerbach, Phys. Rev. B52, 10114 (1995).

[13] A. Onufric and J. B. Marston, Phys. Rev. B 59, 12573(1999).

[14] Y. Q. Li, M. Ma, D. N. Shi and F. C. Zhang, Phys. Rev. Lett. 81(16), 3527(1998).

[15] Y. Yamashita, N, Shibata and K. Ueda, Phys. Rev. B 58, 9114(1998).

[16] B. Frischmuth, F. Mila, abd T. M. Rice, (private communication).

[17] V. L. Pokrovskii and G. V. Uimin, Sov. Phys. JETP 34 457 (1972).

[18] B. Sutherland, Phys. Rev. B 12, 3795(1975); Phys. Rev. Lett. 20, 98(1968).

[19] E. Wigner, Phys. Rev. 51, 1069(1937)
[20] L. Faddeev and L. Takhtajan, Phys. Lett. A 80, 375(1981).

[21] e.g. R. Gilmore, Lie groups, Lie algebras and some of their applications, John Wiley \& Sons, New York, 1974.

[22] I. Affleck and E. Lieb, Lett. Math. Phys. 12, 57(1986); I. Affleck, Phys. Rev. B 37, 5186(1988) and private communications.

[23] E. Lieb, T. Schulz and D. Mattis, Ann. Phys. (NY) 16, 407(1961).

[24] H. Bethe, Z. Phys. 75, 205(1931). J. H. Lowenstein, in Recent Advances in Field Theory and Statistical Mechanics, edited by J. Zuber and R. Stora (Elsevier, Amsterdam 1884) p.612.

[25] F. Woynarovich, J. Phys. C 15, 85(1982); ibid., 97(1982).

[26] D. C. Mattis, The Theory of Magnetism I, SpringerVerlag Berlin Heidelberg New York, 1981.

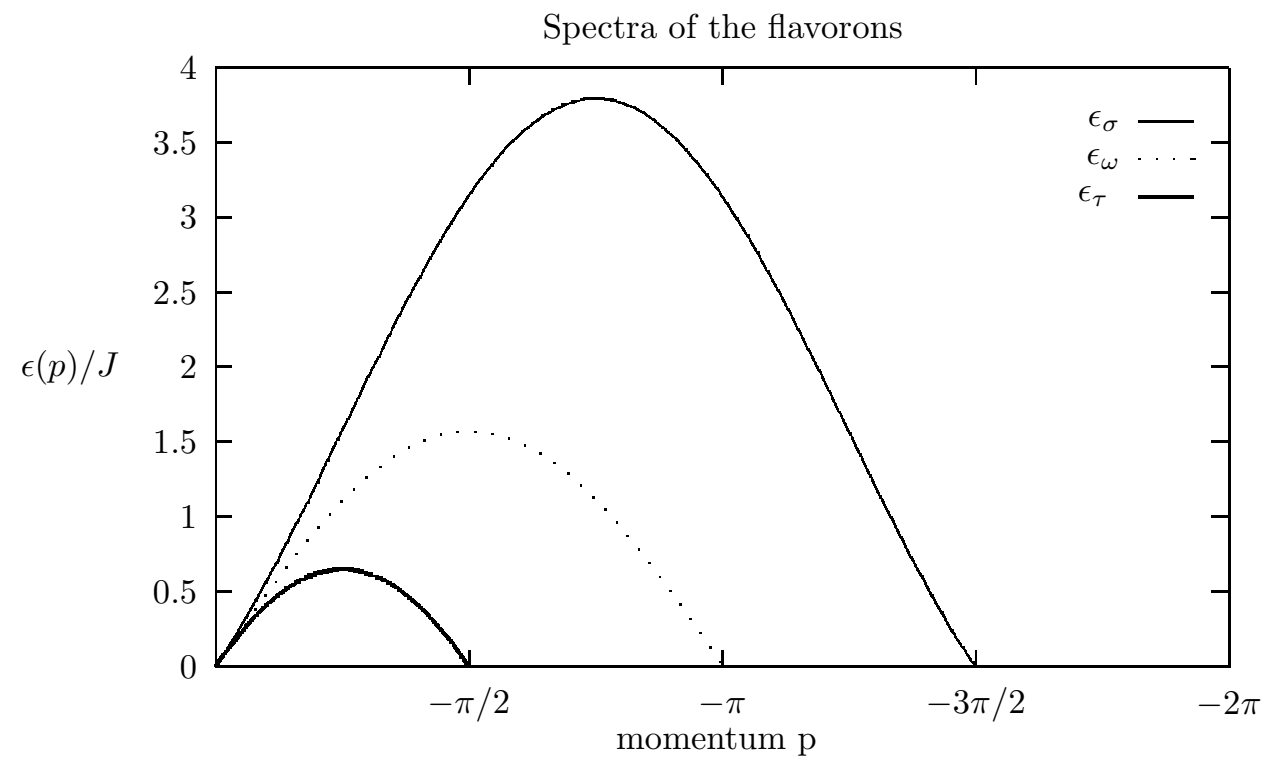

FIG. 1. The spectra of the three type quasi-particles 

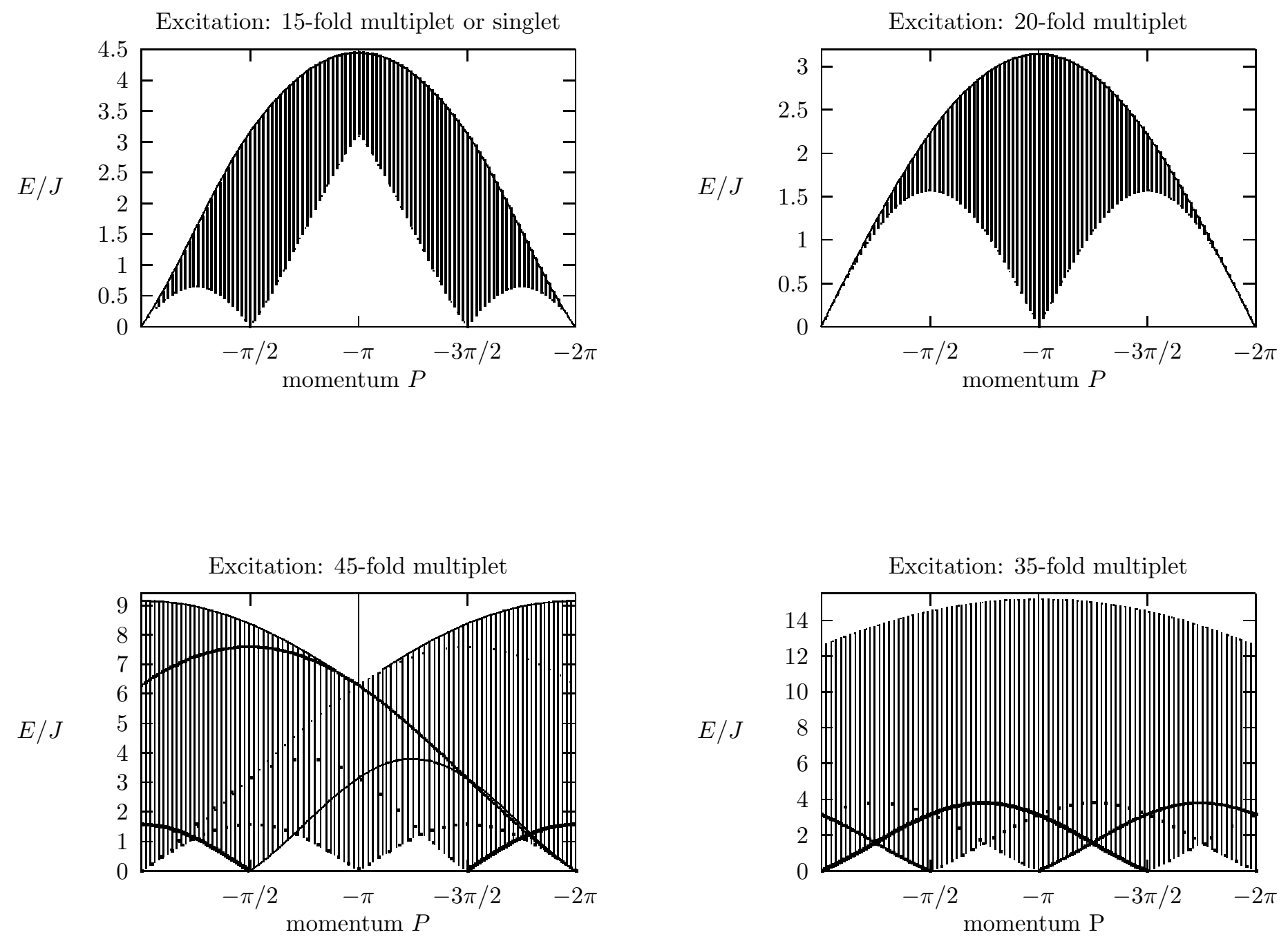

FIG. 2. The dispersions for various types of the generalized magnons. $E$ and $P$ stand for the the corresponding $\Delta E$ and $\Delta P$ in Sec. $\mathrm{VG}$ 


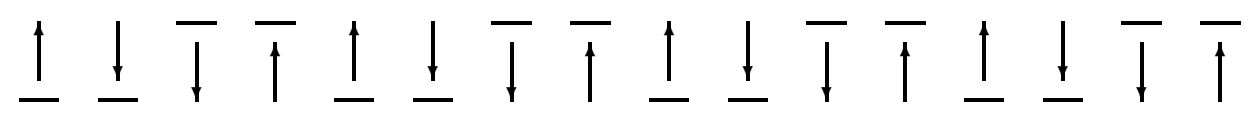

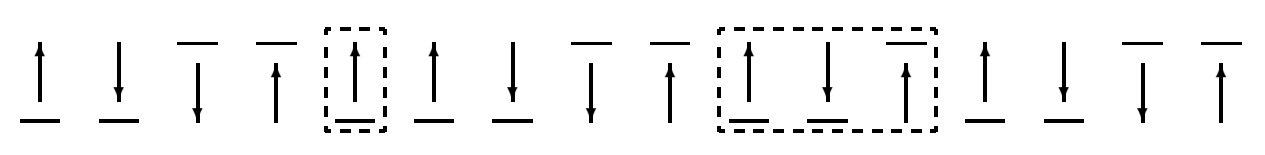

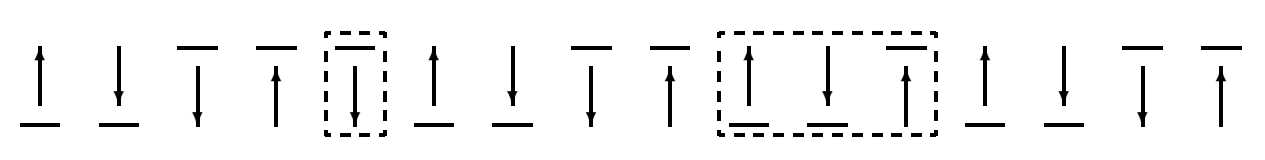

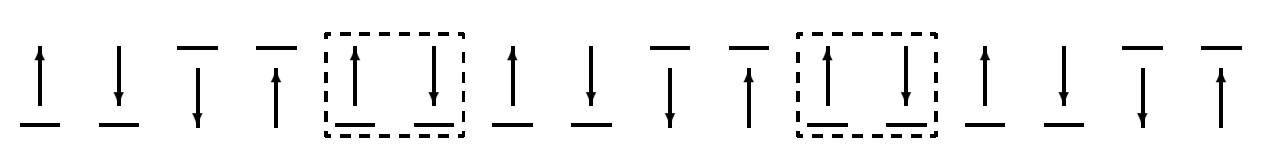

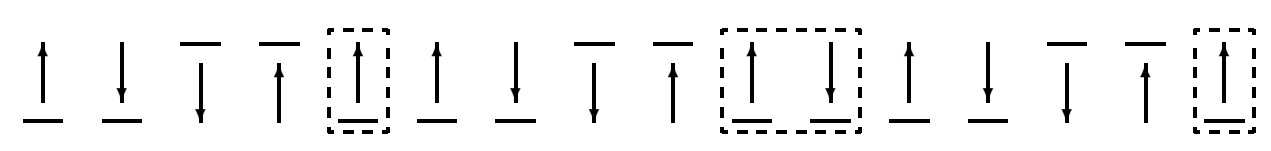

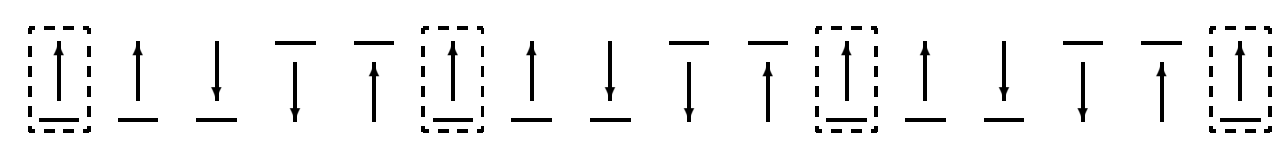

FIG. 3. (a). A $<S_{j} T_{j}>$ staggerily ordered state to demonstrate the ground state, the SU(4) singlet. (b). flavorons of one $\sigma$-type and one $\tau$-type compounded symmetrically to form a 15 -fold multiplet, and (c). compounded anti-symmetrically to form a singlet. (d). The 20-fold multiplet being compounded of two flavorons of $\omega$-type. (e). The 45-fold multiplet being compounded of two flavorons of $\sigma$-type and one flavorons of $\omega$-type. (f). The 35-fold multiplet being compounded of four flavorons of $\sigma$-type. 